Article

\title{
An Improved Method for Reconfiguring and Optimizing Electrical Active Distribution Network Using Evolutionary Particle Swarm Optimization
}

\author{
Nur Faziera Napis ${ }^{1}$, Aida Fazliana Abd. Kadir ${ }^{1}$, Tamer Khatib ${ }^{2, *}$, Elia Erwani Hassan ${ }^{1}$ \\ and Mohamad Fani Sulaima ${ }^{1}$ \\ 1 Faculty of Electrical Engineering, Universiti Teknikal Malaysia Melaka, Malacca 76100, Malaysia; \\ nurfazr@yahoo.com (N.F.N.); fazliana@utem.edu.my (A.F.A.K.); erwani@utem.edu.my (E.E.H.); \\ fani@utem.edu.my (M.F.S.) \\ 2 Department of Energy Engineering, An-Najah National University, Nablus 97300, Palestine \\ * Correspondence: t.khatib@najah.edu; Tel.: +970-5-9931-7172
}

Received: 22 April 2018; Accepted: 14 May 2018; Published: 16 May 2018

\begin{abstract}
The presence of optimized distributed generation (DG) with suitable distribution network reconfiguration (DNR) in the electrical distribution network has an advantage for voltage support, power losses reduction, deferment of new transmission line and distribution structure and system stability improvement. However, installation of a DG unit at non-optimal size with non-optimal DNR may lead to higher power losses, power quality problem, voltage instability and incremental of operational cost. Thus, an appropriate DG and DNR planning are essential and are considered as an objective of this research. An effective heuristic optimization technique named as improved evolutionary particle swarm optimization (IEPSO) is proposed in this research. The objective function is formulated to minimize the total power losses (TPL) and to improve the voltage stability index (VSI). The voltage stability index is determined for three load demand levels namely light load, nominal load, and heavy load with proper optimal DNR and DG sizing. The performance of the proposed technique is compared with other optimization techniques, namely particle swarm optimization (PSO) and iteration particle swarm optimization (IPSO). Four case studies on IEEE 33-bus and IEEE 69-bus distribution systems have been conducted to validate the effectiveness of the proposed IEPSO. The optimization results show that, the best achievement is done by IEPSO technique with power losses reduction up to $79.26 \%$, and $58.41 \%$ improvement in the voltage stability index. Moreover, IEPSO has the fastest computational time for all load conditions as compared to other algorithms.
\end{abstract}

Keywords: distribution network reconfiguration; distributed generation; improved evolutionary particle swarm optimization; voltage stability index

\section{Introduction}

In general, power systems are designed to work with unidirectional power flow due to the lack of power source, except for the main generating power plants [1]. In the meanwhile, the introduction of distributed generation (DG) has changed the fundamental way of the operation in the power system distribution network from passive to active network [2]. Distributed generation can effectively functioning in the distribution system in order to give additional support to the main grid by satisfying load demand, enhancing voltage profile, improving reliability and reducing power losses [3]. The reduction of power losses is very important to maintain the efficiency of the distribution system. There are several approaches to overcome the power losses problems such as network reconfiguration as well as capacitor and DG installation. However, the benefits of these approaches can be achieved if they are carefully coordinated in the distribution system [4]. By examining the literature, it seems that 
only a few approaches have been proposed to take care of this issue. Research in [5-7], for example, have used DG approach only for mitigating voltage stability problem without considering other alternatives such as network reconfiguration.

In general meta-heuristic based optimization techniques are the most prominent method for solving the coordination problems due to its robustness and simplicity [8]. There are many examples of these methods for such an optimization problem. These methods include simulated annealing (SA), particle swarm optimization (PSO), firefly algorithm (FA), artificial immune system (AIS), harmony search algorithm (HAS) and artificial bee colony (ABC). Furthermore, hybridized optimization methods are also proposed by combining two optimization techniques [8]. Among these optimization methods, PSO has become very popular, due to its simplicity and its affinity towards manufacturing and robotics, electrical power systems, engineering and other areas [9]. Therefore, this paper focuses on swarm optimization as a selected algorithm to solve the problem regarding power losses and voltage stability.

In classical PSO cases, PSO sometimes fail to find a global optimum solution. The problem occurs due to the fact that the particles are trapped at a local optimum solution [9]. Therefore, this paper proposes a new optimization algorithm known as improved evolutionary particle swarm optimization (IEPSO) to handle simultaneous approach (distribution network reconfiguration (DNR) and DG) for power loss reduction and to alleviate the problem of voltage stability as well.

This paper is organized as follows, introduction and a section that shows the optimization process of the active distribution networks concept including objective functions and constrains (Section 2). After that, a section that explains the proposed optimization problem is provided. Following that the optimization process is discussed in a separated section (Section 4) and then a detailed results section is provided (Section 5) followed by a conclusion section.

\section{Optimization of Active Distribution Networks for Voltage Stability Improvement and Power Loss Reduction}

The decline of voltage stability is one of the important factors which restrict the increase of load served by distribution networks [10]. Therefore, it is necessary to analyze voltage stability with the variation of load levels in distribution networks that includes DG unit.

In [8] the authors have suggested that voltage stability should be taken as an objective function when addressing the optimal placement of DG units. In addition, two notable studies $[6,11]$ formulated approaches that can be utilised to identify effective placements for DG units to heighten the voltage profile and voltage stability of distribution network.

Voltage instability in distribution systems has been understood for decades and was referred to as load instability [12]. In addition, the effect of DG technology on the improvement of voltage stability in the distribution network is studied by [13-18]. Similar research, such as that conducted in [5], notably examines the impact that a DG unit's capacity and location has on the improvement of voltage stability in distribution networks. In [19] the authors conducted a pragmatic examination of the effect that DG units has on systems stability.

Voltage stability index (VSI) is usually used for assessing load bus stability in a power system, and it can be formulated as follows [20],

$$
L_{i+1}=\frac{4\left[V_{i}^{2}\left(P_{i+1} \times r_{i}+Q_{i+1} \times x_{i}\right)+\left(P_{i+1} \times x_{i}-Q_{i+1} \times r_{i}\right)^{2}\right]}{V_{i}^{4}},
$$

where $L$ is a stability index, $r_{i}+j x_{i}$ is a branch with impedance and it is connected between sending end bus with voltage of $V_{i}$ and receiving node with loading of $P_{i+1}+j Q_{i+1}$.

A modified voltage stability index formula is also given by [21] as follows,

$$
L=4\left[\left(P_{l e q} \times r_{e q}+Q_{l e q} \times x_{e q}\right)+\left(P_{l e q} \times x_{e q}-Q_{l e q} \times r_{e q}\right)^{2}\right]
$$


where $L$ is a stability index, $P_{l e q}+j Q_{l e q}$ is the total real and reactive power respectively and $r_{e q}+j x_{e q}$ is the impedance of the connecting branches of network and they are given as:

$$
\begin{aligned}
& r_{e q}=\sum P_{\text {loss }} /\left[\left(P_{\text {leq }}+\sum P_{\text {loss }}\right)^{2}+\left(Q_{\text {leq }}+\sum Q_{\text {loss }}\right)^{2}\right], \\
& x_{\text {eq }}=\sum Q_{\text {loss }} /\left[\left(P_{\text {leq }}+\sum P_{\text {loss }}\right)^{2}+\left(Q_{\text {leq }}+\sum Q_{\text {loss }}\right)^{2}\right] .
\end{aligned}
$$

For a stable system, the value of stability index, $L$ should be less than 1.0.

Additional formula of VSI is proposed by Shin et al. in [22] for a simple power system with two busses. Voltage stability index was applied in the context of an expansive system incorporating numerous busses. The VSI is formulated in order to evaluate voltage stability in the general radial distribution network as follows,

$$
\mathrm{VSI}=0.5 \times V_{1}-\frac{\left(P_{l e q} \times r_{e q}+Q_{l e q} \times x_{e q}\right)}{V_{1}},
$$

where $V_{1}$ is the sending end voltage of the reduced single line network.

Different studies have utilized different optimization approaches to determine the optimal location of DG units and size with regards to voltage stability and loss reduction [23-26].

The range of the VSI, between unity at no load and zero with regard to the voltage collapse point, can be determined with Equation (15) for line $l$ or for node $m$ as displayed in Figure 1.

$$
\operatorname{VSI}(m)=V_{k}^{4}-4\left\{P_{k m} x_{k m}-Q_{k m} r_{k m}\right\}^{2}-4\left\{P_{k m} r_{k m}-Q_{k m} x_{k m}\right\} V_{k}^{2},
$$

where, $V_{k}$ denotes the voltage magnitude at node- $k, P_{k m}$ represents the sum of real power loads of all the nodes beyond node- $m$ plus the real power load on node- $m$ itself plus the sum of the real power losses of all the branches beyond node- $m, Q_{k m}$ denotes the sum of reactive power loads of all the nodes beyond node- $m$ plus the reactive power load on node- $m$ itself, plus the sum of the reactive power losses of all the branches beyond node- $m, r_{k m}-j x_{k m}$ represents the resistance and reactance of line- $l$, while $P_{m}+j Q_{m}$ represents the real and reactive power load at node- $m$.

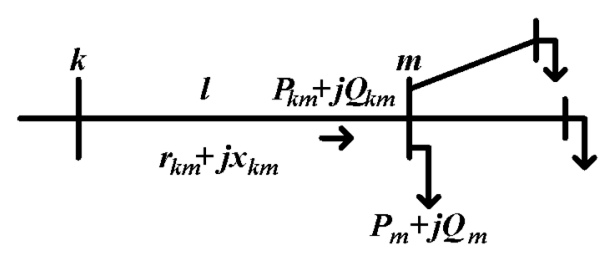

Figure 1. Sample distribution line. $P_{k m}$ represents the sum of real power loads of all the nodes beyond node- $m$ plus the real power load on node- $m$ itself plus the sum of the real power losses of all the branches beyond node- $m, Q_{k m}$ denotes the sum of reactive power loads of all the nodes beyond node- $m$ plus the reactive power load on node- $m$ itself, plus the sum of the reactive power losses of all the branches beyond node- $m, r_{k m}-j x_{k m}$ represents the resistance and reactance of line- $l$, while $P_{m}+j Q_{m}$ represents the real and reactive power load at node- $m$.

On the other hand, the power losses at each line and total power losses can be determined as in Equations (7) and (8), respectively.

$$
\begin{gathered}
\text { Plineloss }_{i j}=\left|I_{i j}\right|^{2} \times R_{i j}, \\
\text { PTotalloss }_{i j}=\sum_{i=1}^{n b r}\left|I_{i}\right|^{2} \times R_{i},
\end{gathered}
$$


where $n b r$ is total number of line, $R_{i j}$ is resistance of the line section between busses $i$ and $j$, and $R_{i}$ is resistance at bus $i$.

The complementary between DG connection and the optimization methods in configuring the network system to achieve greater power loss reduction and best DG sizing are done by changing the power flow direction through the entire network. The objective function of this work is illustrated as below,

$$
f_{\min }=\sum_{\min } P_{\text {Totalloss }}
$$

In this research five constraints are taken into account for the optimization process to ensure all parameters in the distribution network are within allowable limits. The lists of constraints are shown as follows:

(a) Distributed generation operation constraint (continuous variable):

Every DG unit must function in the range of the tolerable value where $P_{D G, \min }$ and $P_{D G, \max }$ which are minimum and maximum limit of DG output power respectively. It is imperative that DG size must not exceed this constraint as shown in Equation (10)

$$
P_{D G, \min } \leq P_{D G} \leq P_{D G, \max }
$$

(b) Bus voltage constraint:

Every voltage value for each bus in the network must function in the range of the permissible bound where $V_{n, \min }$ and $V_{n, \max }$ are the minimum and maximum allowable voltage at bus $n$ respectively as shown in the equation below,

$$
V_{n, \min } \leq V_{n} \leq V_{n, \max }
$$

(c) Total power injection constraint:

In order to avoid the power injection from the DG units to the main grid (substation), the total power output of DG must be less than the total load $\left(P_{l o a d}\right)$ on the network and total power losses $\left(P_{\text {losses }}\right)$. Hence, it is possible to avoid the reverse power flow as shown below,

$$
\sum_{k=1}^{t d g} P_{D G, k}<\sum_{n=1}^{t l} P_{l o a d, n}+\sum_{i=1}^{n b r}\left|I_{i}\right|^{2} \times R_{i}
$$

where the $t d g$ and $t l$ denote the total number of DG units and the total number of loads respectively. $P_{D G}$ denotes the size of the DG and $P_{\text {load }}$ that represents the total number of loads consumed at bus $n$.

(d) Power balanced constraint:

The total power injected into the system which is from the DG unit and main substation, $P_{\text {substation }}$ must be equal with total load and the total power loss (power equilibrium concept) as illustrated in the following equation.

$$
\sum_{k=1}^{t d g} P_{D G, k}+P_{\text {substation }}=\sum_{n=1}^{t l} P_{\text {load }, n}+\sum_{i=1}^{n b r}\left|I_{i}\right|^{2} \times R_{i}
$$

(e) Radial configuration constraint:

Finally, in order to eliminate the excessive of current flow in the system, this network configuration must be in radial. Hence, a range of constraints must be factored into account if the radial network is to be sustained in a consistent manner. Several standard rules have been adopted for selection of switches. Those switches that do not belong to any loop, connected to the sources and contributed to a meshed network have to be closed. 


\section{Proposed Improved Evolutionary Particle Swarm Optimization for Optimization of Active Distributed Network}

In this paper, three optimization techniques are considered to optimize active distribution network for voltage stability improvement and power loss reduction which are PSO, IPSO and IEPSO.

\subsection{Particle Swarm Optimization}

The main idea of PSO is based on the food searching behavior (foraging) of birds or fish that move towards food at certain speeds and positions. Their movement depends on their own experience and experience from other 'friends' in the group $\left(P_{b e s t}\right.$ and $\left.G_{b e s t}\right)$. The new velocity, $v_{i}+1$ and the new position, $x_{i}+1$ for the fishes or birds are obtained using Equations (14) and (15) after the current $G_{b e s t}$ and $P_{\text {best }}$ have been calculated [13].

$$
\begin{gathered}
v_{i+1}=\omega V_{i}+c_{1} r_{1}\left(P_{\text {best }}-x_{i}\right)+c_{2} r_{2}\left(G_{\text {best }}-X_{i}\right), \\
x_{i+1}=v_{i+1}+X_{i},
\end{gathered}
$$

where $r_{1}$ and $r_{2}$ are two random variables in the range zero to one, $c_{1}$ and $c_{2}$ are positive constants, $\omega$ is the inertia weight. $X_{i}=\left(x_{i}^{1}, x_{i}^{2}, \ldots, x_{i}^{n}\right)$ and $V_{i}=\left(v_{i}^{1}, v_{i}^{2}, \ldots, v_{i}^{n}\right)$ represent the position and velocity of the $i$ th particle, respectively. $P_{\text {best }}$ represents the personal best location of $i$ th particle. $G_{b e s t}$ represents the global best location of the whole swarm which can be expressed as follows,

$$
\begin{aligned}
& X^{\text {Pbest }}=\left\{\begin{array}{ll}
X^{\text {Pbest }(j)} & \text { if } F V^{j+1} \geq F V^{j} \\
X_{i}^{t} & \text { if } F V^{j+1} \leq F V^{j}
\end{array},\right. \\
& X^{\text {Gbest }}= \begin{cases}X^{\text {Gbest }(j)} & \text { if } F V^{j+1} \geq F V^{j} \\
X^{\text {Pbest }(j+1)} & \text { if } F V^{j+1} \leq F V^{j}\end{cases}
\end{aligned}
$$

where $F V$ is fitness value.

The optimization process of PSO algorithm can be listed in points as follows

Step 1: Initialization: This stage provides the configuration of the distribution network and candidate DG sizing and location. By establishing the random initial population and the number of iterations, the functional model is generated. Other elements drawn on are the random initial selection of position and velocity in the context of the search space.

Step 2: Calculate fitness $(f(x))$ : The network solution program is set up with the initial data whereby the fitness will calculate the summation of each particle.

Step 3: Find $P_{\text {best }}$ and $G_{\text {best }}$ for all populations:

- $\quad$ Finding $P_{\text {best }}$ : The parameters of fitness values associated with the placement of each particle are considered in relation to the matching value in previously held positions. Following this, the lower fitness value is listed as $P_{\text {best }}$ in the current iteration.

- $\quad$ Finding $G_{\text {best }}$ : Here, the lowest fitness for $P_{\text {best }}$ is associated with every particle in the current iteration. It is then compared to the previous iteration and the lower one is recorded as the $G_{\text {best }}$.

Step 4: Calculate the new velocity for each population. The position and velocity of particles can be calculated for next iteration.

Step 5: Adjust the position (new position, $x_{\text {new }}$ ). Here, the value is updated.

Step 6: If the specification is not satisfied, meaning the most minimum value, then, back to Step 2.

Step 7: The final output is the value of $G_{b s e t}$. 


\subsection{Iteration Particle Swarm Optimization}

Iteration particle swarm optimization (IPSO) is a modification version of PSO method which proposed by [27]. Iteration particle swarm optimization offers the improvement of quality solution and computational time of the algorithm. In the IPSO, three best values were used to update the velocity and the position of the particles, which are known as $P_{b e s t}, G_{b e s t}$ and $I_{b e s t}$. The definition and the method to determine the $P_{\text {best }}$ and $G_{\text {best }}$ values in the IPSO are similar to the original PSO. Meanwhile, the new parameters $I_{\text {best }}$ is defined as the best value of the fitness function that has been achieved by any particle in the present iteration. In other words, $I_{\text {best }}$ value is the $P_{\text {best }}$ value that is randomly selected among the existing particles from the current population. In addition, the authors also introduced a dynamic acceleration constant parameter, $c_{3}$ value for $I_{\text {best }}$ in IPSO. Therefore, the new velocity formula for the IPSO algorithm is shown in Equation $(18)[27,28]$.

$$
\begin{gathered}
V_{i+1}=w v_{i}+c_{1} r_{1}\left(P_{\text {best }}-x_{i}\right)+c_{2} r_{2}\left(G_{\text {best }}-x_{i}\right)+c_{3}\left(I_{\text {best }}-x_{i}\right), \\
c_{3}=c_{1}\left(1-e^{-c_{1} k}\right),
\end{gathered}
$$

where $r_{1}$ and $r_{2}$ are two random variables in the range zero to one, $c_{1}$ and $c_{2}$ are positive constants, $c_{3}$ is the acceleration constant that pulls each particle towards $I_{\text {best }}$. The value of $c_{3}$ will change according to $c_{1}$ and the number of iteration $k, \omega$ is the inertia weight. $X_{i}=\left(x_{i}^{1}, x_{i}^{2}, \ldots, x_{i}^{n}\right)$ and $V_{i}=\left(v_{i}^{1}, v_{i}^{2}, \ldots, v_{i}^{n}\right)$ represent the position and velocity of the $i$ th particle, respectively. $P_{b e s t}$ represents the personal best location of $i$ th particle. $G_{\text {best }}$ represents the global best location of the whole swarm. $I_{b e s t}$ represents one value taken from the personal best location of $i$ th particle.

\subsection{Improved Evolutionary Particle Swarm Optimization}

As mentioned earlier, PSO sometimes fails to find a global optimum solution. This problem occurs due to the fact that the particles are trapped at a local optimum (premature) solution. There are three main constant parameters that will affect the performance of the PSO, which are inertia weight $(w)$, cognitive constant $\left(c_{1}\right)$, and social constant $\left(c_{2}\right)$; the changes of these parameters will result in different performances of the algorithm. In a traditional PSO, these parameters are unspecified and need to be adjust several times in order to obtain a suitable value. This implies that there are possibilities for the user to obtain inaccurate settings on the initial values $\left(c_{1}, c_{2}\right.$, and $\left.w\right)$ in PSO, which leads to unconverged solution.

Based on the weakness of the conventional PSO, an improved evolutionary PSO is proposed in this paper which aims to improve the solution quality and computing time of the algorithm. In the proposed IEPSO, evolutionary programming (EP) is applied due to its excellent capability for searching optimal solution in a complex problem. Evolutionary programming utilizes selection processes that are based on programs referred to as "tournament schemes", and these are employed in order to select the survivals for the successive generation. This approach is highly effective in identifying the optimal candidates that can be conveyed into the successive generation of the combined population of the parents of the offspring. Therefore it is superior is compared to PSO and IPSO in term of solution quality and computing time in solving problem. Following the tournament and selection process, the position is categorized in descending fashion. This begins with the top score and proceeds down to the lowest, thus the name. The position with the highest score is employed as the novel $G_{b e s t}$ while all other positions are utilized as the newest $P_{\text {best }}$. Following this, certain values are chosen in a random manner within the $P_{\text {best }}$ in order to function as the new $I_{\text {best }}$ as a new velocity that corresponds to the fitness value. Figure 2 shows a the optimization process of IEPSO. 


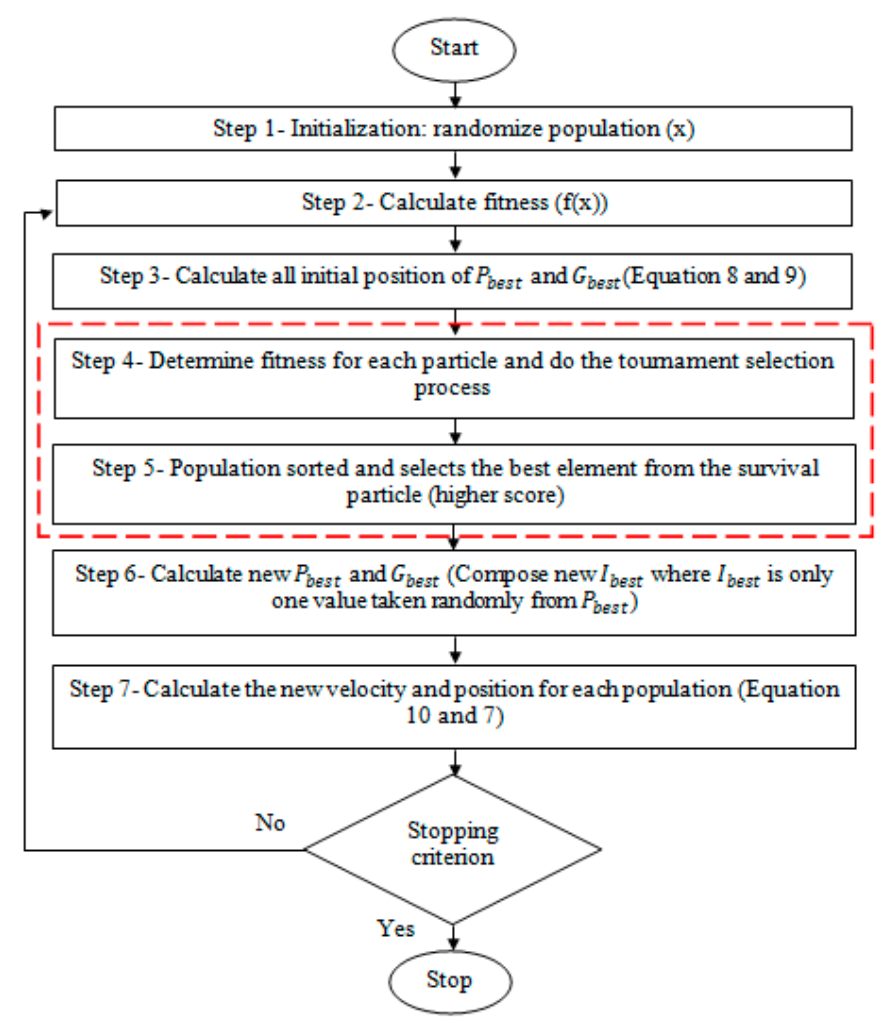

Figure 2. Improved evolutionary particle swarm optimization (IEPSO) algorithm flowchart.

\section{Distributed Generation Placement Procedure}

The potential buses for DG placement can be selected randomly, by recommended location or depending on the results of voltage profile. For this study, the location of DG is selected based on the sensitive bus that closer to voltage collapse. Figure 3 shows the determination process of DG unit placement in the system.

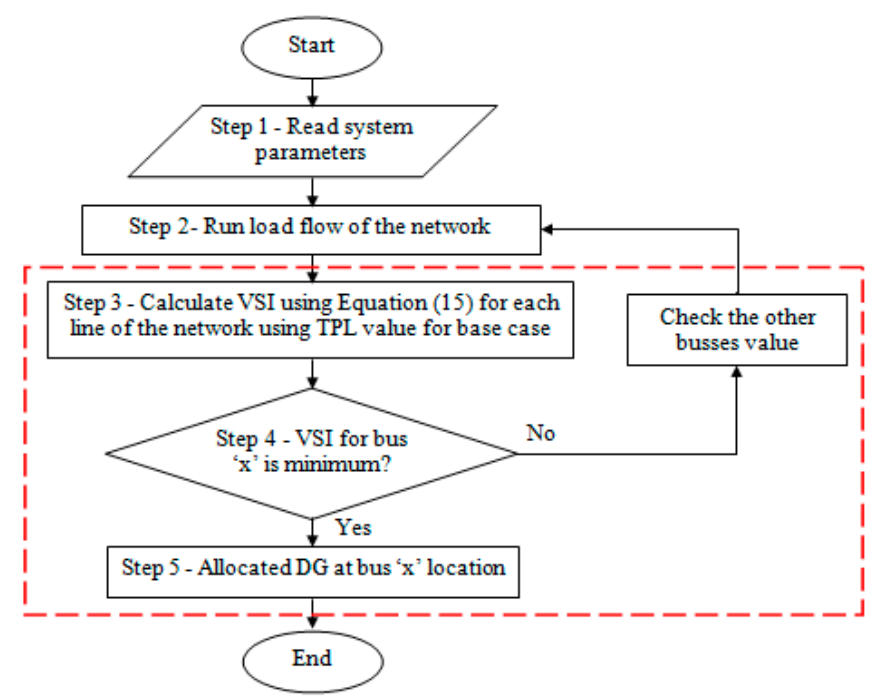

Figure 3. Process to allocate the distributed generation (DG). VSI: voltage stability index; TPL: total power losses.

The optimal size of DG unit is obtained from the simulation in which both parameters DG (represented by $P_{g}$ ) and the switches opened (represented by $S_{m}$ ) are automatically adjusted during 
simulation. Tie switch and sectionalizing switch together with the DG size are considered as the main control variables. The implementation of the proposed methods for solving the DNR and DG sizing problem simultaneously are developed in MATLAB (Mathworks, Natick, MA, USA, Version Matlab 2016) environment and can be described as follows and shown in Figure 4.

(i) Initialization:

First, in the MATLAB environment, all the input system data, such as network data, busses data, line data, predefined range of DG size, maximum iteration and voltage limit are inserted in order to run the optimization algorithm. The initialization population is determined by selecting tie switches from the set of original tie switches. Those variables are generated by the system via a random generator available in the program and they are utilized to compute the power losses in the next step. In this work, the particles consist of tie line $(S)$ and DG size $\left(P_{g}\right)$ as shown in Equation (20) proposed as particles.

$$
X_{\text {particle }}=\left\{S_{1}, S_{2}, \ldots, \ldots S_{\beta}, P_{g 1}, P_{g 2}, \ldots, \ldots P_{g \alpha}\right\},
$$

where, $\beta$ is the number of tie line, and $\alpha$ is the number of DG. Only the particles that satisfy all the constraints are considered as the initial population.

This step involves initializing the following IEPSO parameters: the number of particles, $N$, the weighing factors, $C_{1}, C_{2}$ and $C_{3}$ and finally, the maximum number of iterations. In an attempt to ensure the maintenance of the radial network, the system must take into account the bounds. Three rules in constraints (e) have been utilized for the switch choices.

(ii) Fitness calculation:

In this step, an initial population of particles with random position $X$ and velocities $V$ on dimension in the solution space is randomly generated. For each particle that fulfills the constraints as mentioned in constraints (a) until (e), the power flow is accomplished and the total power loss has been obtain through the Newton-Raphson load flow program.

(iii) Determination of $P_{\text {best }}, G_{\text {best }}$ and $I_{\text {best }}$ :

During the searching process, the two values as the best values are updated and recorded. This value is noted as $P_{\text {best }}$ and another best value to be verified is $G_{b e s t}$, which the whole best value so far by any particle. The $P_{\text {best }}$ and $G_{\text {best }}$ represent the generation of tie-switches and total power loss. While $I_{\text {best }}$ is a random value taken from $P_{\text {best }}$ to be added to the new velocity calculated afterwards.

(iv) New velocity and position:

The particle's velocity and position are updated. The particle's velocity signifies a switches movement. Meanwhile, the total power loss of all switches is evaluated by using the new position $X_{\text {new }}$.

(v) Combination and tournament selection process:

After obtaining the new position $X_{n e w}$, the new fitness value (total power loss) is obtained using the value of new positions. Thus, the set of new position $X_{\text {new }}$ and the old set position $X$ are combined together. This combination of new and old set position is contested in a tournament. A position $X$ wins when its fitness is better than other contenders and this tournament is contested as randomly. The selection strategy in this process is done using priority selection strategy. In this technique, the old set position $X$ and the new position $X_{\text {new }}$ are sorted in descending order according to total power loss in the distribution system.

(vi) Convergence test:

The new position set will be tested for convergence. After some iteration, the fitness value of the population would be the same. If convergence is not achieved, the process is repeated from the step (ii) until (v). If convergence is achieved, then the optimization process is terminated. 
(vii) Voltage stability index min calculation:

System's VSI min is computed here. This is attributed to the conveyance of power at critical points through the distribution line.

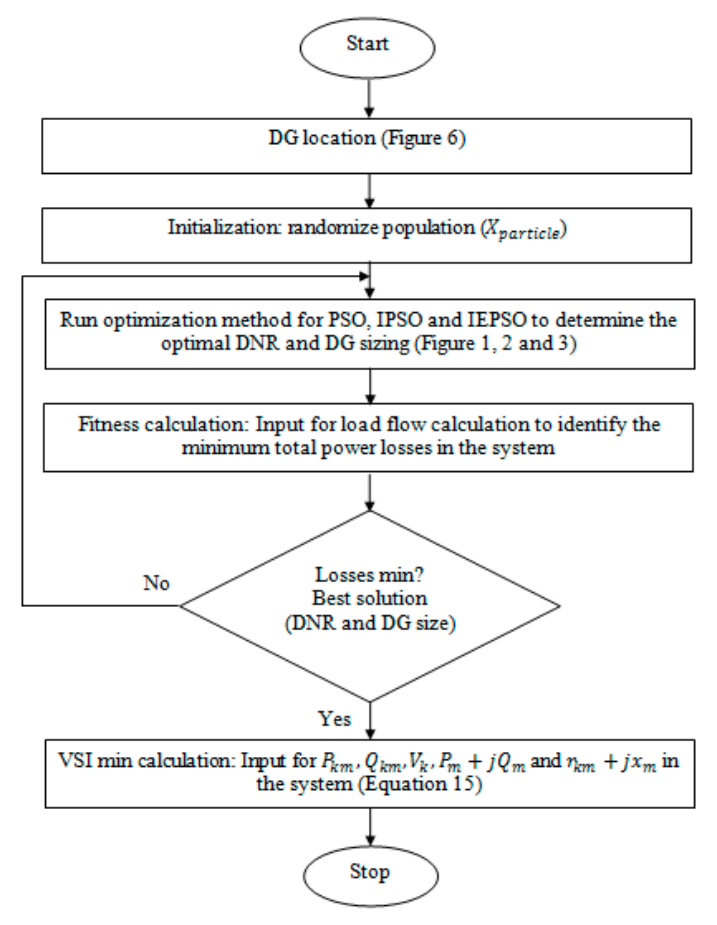

Figure 4. Overall process for distribution network reconfiguration (DNR) and DG sizing.

\section{Results and Discussion}

In this research two test systems are used to evaluate the performance of the proposed algorithm which are the 33-bus radial distribution system and the 69-bus radial distribution systems along with different load levels were utilized.

\subsection{Results of 33-Bus Radial Distribution System}

The 33-bus radial distribution system consists of a feeder, 5 tie switches. The total nominal load of this system is $3715 \mathrm{~kW}$ and $2300 \mathrm{kVAr}$. On the other hand, the 69-bus radial distribution system consists of a feeder and involves 5 tie switches. The total nominal load system is $3802 \mathrm{~kW}$ and $2695 \mathrm{kVAr}$. Both systems MVA base has a base power constant, $S_{\text {base }}$ of 100 MVA and base voltage $V_{\text {base }}$ of $12.66 \mathrm{kV}$. The maximum active output of DG in this study is set at $50 \%$ of the connected load. The size of a population for test systems is assumed to be 20, while, the convergence value is taken as 0.0001 .

The analysis of the results has been divided into three main parts, which are: (i) determination of DG location; (ii) minimization of total power losses (TPL) and VSI improvement with DNR implementation; and (iii) minimization of TPL and VSI improvement with DNR and DG implementation. Three situations have been considered in this study, which are base case, DNR, and DNR with DG. Three load levels are classified into; light, nominal and heavy load in order to evaluate the performance of TPL and VSI respectively. Each situation except for base case has been analyzed among PSO, IPSO and IEPSO algorithms.

Initially, the DG location has been set up according to the bus with minimum VSI. The minimum VSI means the most sensitive probability to voltage collapse. The DG is assumed to operate in PQ mode, where the power output is constant. Then, the stability index, $L$ is calculated to verify the results obtained as shown in Table 1. 
Table 1. The determination of DG bus location.

\begin{tabular}{ccccccc}
\hline \multirow{2}{*}{ Test System } & \multicolumn{2}{c}{ 33-Bus Radial Distribution System } & \multicolumn{2}{c}{ 69-Bus Radial Distribution System } \\
\cline { 2 - 7 } & $\begin{array}{c}\mathbf{0 . 5} \text { (Light } \\
\text { Load) }\end{array}$ & $\begin{array}{c}\mathbf{1 . 0} \text { (Nominal } \\
\text { Load) }\end{array}$ & $\begin{array}{c}\mathbf{1 . 5} \text { (Heavy } \\
\text { Load) }\end{array}$ & $\begin{array}{c}\mathbf{0 . 5} \text { (Light } \\
\text { Load) }\end{array}$ & $\begin{array}{c}\mathbf{1 . 0} \text { (Nominal } \\
\text { Load) }\end{array}$ & $\begin{array}{c}\mathbf{1 . 5} \text { (Heavy } \\
\text { Load) }\end{array}$ \\
\hline Stability Index $(L)$ & 0.0979 & 0.1995 & 0.3056 & 0.0917 & 0.1851 & 0.2979 \\
VSI min Base & 0.8443 & 0.6970 & 0.5583 & 0.8351 & 0.6799 & 0.5321 \\
DG Location & 17 & 17 & 17 & 65 & 65 & 65 \\
\hline \multicolumn{7}{c}{ VSI: voltage stability index } \\
\hline
\end{tabular}

In this research, there are three categories to be analyzed namely base case, DNR and DNR with DG sizing. The analysis is discussed according to three identified load levels as light, nominal and heavy load. Thus, the performances are evaluated among case 1 (base case), case 2 (PSO), case 3 (IPSO) and case 4 (IEPSO).

Table 2 shows base case results without reconfiguration and DG placement (case 1). From the table, the VSI values are inversely proportional with TPL for each possibility load levels. In other words, by increasing the load levels, the total loss has significantly increased and the stability is decreased.

Table 2. Base case at different load conditions for 33-bus system.

\begin{tabular}{ccccc}
\hline & & \multicolumn{3}{c}{ Load Levels } \\
\cline { 3 - 5 } & & Light Load & Nominal Load & Heavy Load \\
\hline \multirow{2}{*}{ Case 1: Before } & Switches & $33,34,35,36,37$ & $33,34,35,36,37$ & $33,34,35,36,37$ \\
reconfiguration & TPL (kW) & 47.06 & 202.62 & 496.19 \\
(Base Case) & VSI min & 0.8443 & 0.6970 & 0.5583 \\
& Voltage Profile min (p.u.) & 0.9582 & 0.91312 & 0.86348 \\
\hline
\end{tabular}

On the other hand, Table 3 summarizes the performance of three optimization techniques for DNR switches, the TPL, VSI, the minimum bus voltage profile and computational time for these three different load levels. From the result obtained in Table 3, the IEPSO technique provided the best optimal solution with the lowest power losses, improved VSI and less computational time as indicated in bold among all load conditions. From the results, IEPSO technique offered the most completion reduction with $4.16 \%$ for TPL, the fastest in computational time, $2.62 \%$ highest VSI in light load among others with identified methods.

Table 3. The performance of particle swarm optimization (PSO), improved particle swarm optimization (IPSO) and IEPSO for DNR implementation in 33-bus radial distribution system.

\begin{tabular}{ccccc}
\hline \multirow{2}{*}{ Load Levels } & Parameters & \multicolumn{3}{c}{ DNR } \\
\cline { 2 - 4 } & & Case 2: PSO & Case 3: IPSO & Case 4: IEPSO \\
\hline \multirow{4}{*}{ Light Load } & Switches & $28,7,10,17,33$ & $24,10,18,8,7$ & $7,12,10,31,26$ \\
& TPL (kW) & 45.9 & 45.4 & 45.1 \\
& VSI min & 0.8622 & 0.8657 & 0.8664 \\
& Voltage profile (p.u.) & 0.9636 & 0.9646 & 0.9646 \\
& Computational time (s) & 30.044280 & 4.545669 & 2.055129 \\
\hline \multirow{4}{*}{ Nominal Load } & $7,33,34,28,14$ & $28,15,33,8,34$ & $9,13,33,6,26$ \\
& Switches & 167.10 & 158.80 & 155.50 \\
& TPL (kW) & 0.7181 & 0.7216 & 0.7333 \\
& VSI min & 0.9206 & 0.9217 & 0.9254 \\
& Voltage profile (p.u.) & 19.630266 & 1.516081 & 2.216311 \\
\hline \multirow{2}{*}{ Heavy Load } & Computational time (s) & $33,10,7,21,28$ & $14,33,32,9,28$ & $33,11,34,28,32$ \\
& Switches & 391.00 & 342.9 & 340.6 \\
& TPL (kW) & 0.6618 & 0.6718 & 0.6763 \\
& VSI min & 0.9020 & 0.9054 & 0.9069 \\
& Voltage profile (p.u.) & 14.352825 & 4.281991 & 3.445760 \\
\hline
\end{tabular}


In addition to that, tor nominal load, IEPSO technique also gave the most reduction with $23.26 \%$ in TPL, $5.21 \%$ highest VSI, but slightly defeated in computational time compared to IPSO by $0.7 \mathrm{~s}$ in nominal load. Finally, for heavy load, IEPSO technique gave the most reduction with $31.36 \%$ in TPL, $21.14 \%$ highest VSI, the fastest in computational time in heavy load compared to other techniques.

As for the DNR with DG placement case, Table 4 summarizes the performances of three optimization techniques in DNR with DG sizing simultaneously for switches, TPL, VSI, voltage profile, DG sizing and computational time at three different load levels. As noted from Table 4, the IEPSO technique gives the best optimal solution with the lowest power losses, improved VSI and computational time as indicated in bold for all load conditions. The increased in load levels, gave results in increasing power losses and decreasing VSI.

Table 4. The performance of PSO, IPSO and IEPSO for DNR with DG sizing implementation in 33-bus radial distribution system.

\begin{tabular}{lcccc}
\hline \multirow{2}{*}{ Load Levels } & Parameters & \multicolumn{3}{c}{ DNR } \\
\cline { 2 - 4 } & & Case 2: PSO & Case 3: IPSO & Case 4: IEPSO \\
\cline { 2 - 4 } & Switches & $8,3,10,22,14$ & $9,28,16,33,34$ & $33,28,34,16,9$ \\
Light Load & DG sizing (MW) & 1.0139 & 1.0147 & 1.0087 \\
& TPL (kW) & 39.1 & 38.9 & 38.6 \\
& VSI min & 0.9239 & 0.9316 & 0.9316 \\
& Voltage profile min (p.u.) & 0.9688 & 0.9824 & 0.9824 \\
& Computational time (s) & 14.246903 & 2.682595 & 2.191424 \\
\hline \multirow{4}{*}{ Nominal Load } & $7,15,10,33,27$ & $15,8,28,7,11$ & $10,33,25,3,22$ \\
& Switches & 1.1368 & 1.1162 & 1.1290 \\
& DG sizing (MW) & 145.1 & 131.3 & 129.0 \\
& TPL (kW) & 0.7861 & 0.8133 & 0.8236 \\
& VSI min & 0.9398 & 0.9497 & 0.9527 \\
& Voltage profile min (p.u.) & 25.102418 & 2.183219 & 6.634950 \\
\hline \multirow{4}{*}{ Heavy Load } & Computational time (s) & $7,33,9,31$ & $12,15,28,8,6$ & $33,13,27,7,16$ \\
& Switches & 1.0041 & 1.0138 & 1.0363 \\
& DG sizing (MW) & 384.5 & 309.8 & 309.00 \\
& TPL (kW) & 0.6687 & 0.6828 & 0.7159 \\
& VSI min & 0.9015 & 0.9092 & 0.9200 \\
& Voltage profile min (p.u.) & 10.980474 & 2.337904 & 2.254589 \\
\hline
\end{tabular}

For light load, IEPSO technique gave the most reduction with $17.98 \%$ in TPL, $10.34 \%$ highest VSI, the fastest in computational time in light load compared to other techniques. Meanwhile, for nominal load IEPSO technique gave the most reduction with $36.33 \%$ in TPL, $18.16 \%$ highest VSI, but slightly defeated for the computational time compared to IPSO by $4.45 \mathrm{~s}$ in nominal load level. Finally, for heavy load IEPSO technique gave also the most reduction with $37.73 \%$ in TPL, $28.22 \%$ highest VSI, the fastest in computational time in heavy load compared to other techniques.

As for system's stability, Figures 5 and 6 show some improvement in the stability for all cases, especially with proposed algorithm application on 33-bus test system. Generally, by increasing the load levels, the VSI will decreases. Thus, the stability of the system which leads to voltage collapse form the load demands increase.

For comparison purposes, Figure 7 shows a comparison of TPL and VSI for three cases which are base case, DNR without DG and DNR with DG for light, nominal and heavy load condition in 33-bus system. From the figures, it is shown that TPL is slightly reduced with DNR implementation. However, the TPL has a significant reduced when DNR and DG was applied to the system. Moreover, the VSI also improved when DNR and DG are implemented optimally in the system. 


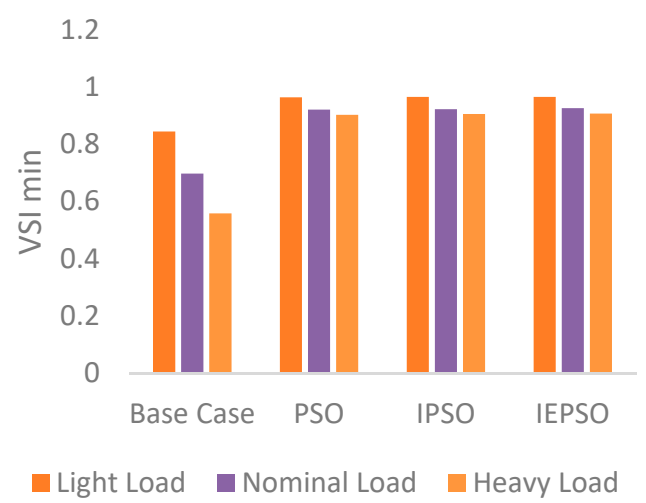

Figure 5. Voltage stability index for different load levels for DNR in 33-bus test system.

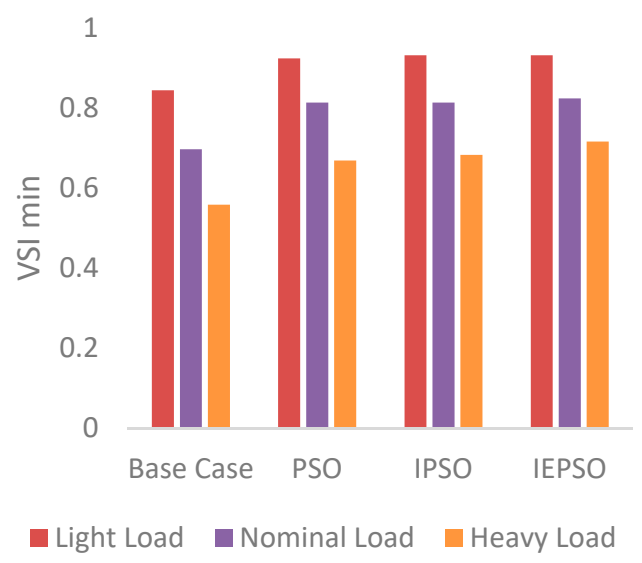

Figure 6. Voltage stability index for different load levels for DNR with DG in 33-bus test system.

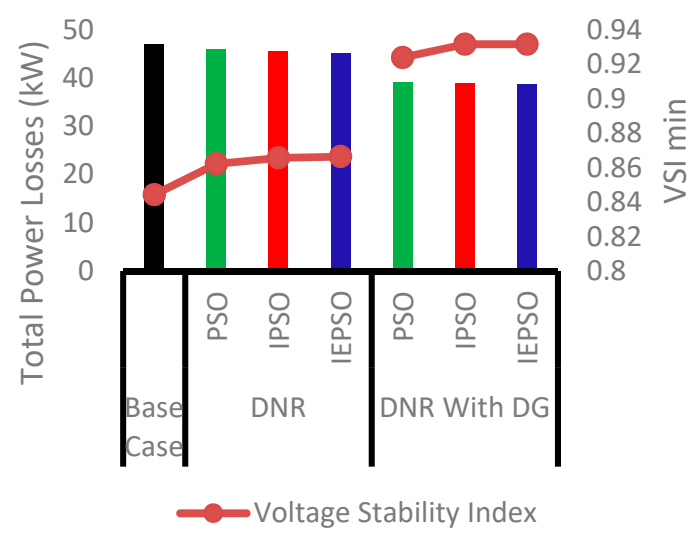

Figure 7. Total power losses and VSI min for DNR without and with DG at light load in 33-bus test system.

In addition, it is observed that during light load of 33-bus test system, the total power loss obtained from the PSO algorithm (case 2) with DG is $39.1 \mathrm{~kW}$ as compared to $45.9 \mathrm{~kW}$ without DG resulted to $14.45 \%$ TPL reduction. The VSI without DG is 0.8622 which is improved to be 0.9239 p.u. with DG integration. For IPSO algorithm (case 3), the total power loss is $38.9 \mathrm{~kW}$ with DG compared to $45.4 \mathrm{~kW}$ without DG with $6.5 \mathrm{~kW}$ differences, with $13.81 \%$ TPL reduction. The VSI without DG is 0.8657 which is improved to be 0.9316 with DG integration. Finally, For IEPSO algorithm (case 4), the total power loss is $38.6 \mathrm{~kW}$ with DG compared to $45.1 \mathrm{~kW}$ without DG with $6.5 \mathrm{~kW}$ differences, with $13.81 \%$ TPL reduction. The VSI without DG is only 0.8664 and enhanced to e 0.9316 with DG integration. 
Similarly, it is observed that at nominal load in 33-bus test system, the total power loss obtained from the PSO algorithm (case 2) with DG is $145.1 \mathrm{~kW}$ compared to $167 \mathrm{~kW}$ without DG with $22 \mathrm{~kW}$ differences, resulted to $10.86 \%$ TPL reduction. The VSI without DG is 0.7181 which is improved to be 0.7861 with DG integration. For IPSO algorithm (case 3), the total power loss is $131.3 \mathrm{~kW}$ with DG compared to be $158.8 \mathrm{~kW}$ without DG with $27.5 \mathrm{~kW}$ differences, with $13.57 \%$ TPL reduction. The VSI without DG is 0.7216 which is improved to be 0.8133 with DG integration. Finally, For IEPSO algorithm (case 4), the total power loss is $129 \mathrm{~kW}$ with DG compared to $155.5 \mathrm{~kW}$ without DG with $26.5 \mathrm{~kW}$ differences, with $13.08 \%$ TPL reduction. The VSI without DG is 0.7333 which is improved to be 0.8236 with DG integration.

As for heavy load in 33-bus test system, the total power loss obtained from the PSO algorithm (case 2) with DG is $384.5 \mathrm{~kW}$ compared to $391 \mathrm{~kW}$ without DG with $6.5 \mathrm{~kW}$ differences, resulted in $1.31 \%$ TPL reduction. The VSI without DG is 0.6618 which is improved to be 0.6687 with DG integration. For IPSO algorithm (case 3), the total power loss is $309.8 \mathrm{~kW}$ with DG compared to $342.9 \mathrm{~kW}$ without DG with $33.1 \mathrm{~kW}$ differences, with $6.67 \%$ TPL reduction. The VSI without DG is 0.6718 which is improved to be 0.6828 with DG integration. For IEPSO algorithm (case 4), the total power loss is $309 \mathrm{~kW}$ with DG compared to $340.6 \mathrm{~kW}$ without DG with $31.6 \mathrm{~kW}$ differences, $6.37 \%$ TPL reduction. The VSI without DG is 0.6763 which is improved to be 0.7159 with DG integration.

\subsection{Results of 69-Bus Radial Distribution System}

Similarly, as before in 33-bus system, there are three parts to be analyzed; base case, DNR and DNR with DG sizing. At each part, the analysis is divided into three different load levels. Meanwhile, the comparison of the effectiveness of the proposed method is compared with the base case, PSO and IPSO.

Table 5 shows the base case without reconfiguration and DG placement. In light load level ( $50 \%$ of nominal load), the total power loss is $53.24 \mathrm{~kW}$, while the minimum VSI is 0.8351 .

Table 5. Base case at different load conditions for 69-bus system.

\begin{tabular}{ccccc}
\hline \multirow{2}{*}{ Cases } & \multicolumn{3}{c}{ Load Levels } \\
\cline { 3 - 5 } & & Light Load & Nominal Load & Heavy Load \\
\hline \multirow{2}{*}{ Case 1: Before } & Switches & $69,70,71,72,73$ & $69,70,71,72,73$ & $69,70,71,72,73$ \\
reconfiguration & TPL (kW) & 53.24 & 229.73 & 573.24 \\
(Base Case) & VSI min & 0.8351 & 0.6799 & 0.5321 \\
& Voltage Profile min (p.u.) & 0.9560 & 0.9081 & 0.8541 \\
\hline
\end{tabular}

For nominal load level, the total power loss is $229.73 \mathrm{~kW}$, with the minimum VSI is 0.6799 . Meanwhile, at heavy load level (150\% of nominal load), the total power loss is $573.24 \mathrm{~kW}$ with minimum VSI is 0.5321 . The VSI value is inversely proportional with total power loss. By increasing the load levels, the total power loss is increased significantly and the system becomes unstable.

On the other hand, Table 6 summarizes the performances of three optimization technique in DNR for switches, TPL, VSI, voltage profile, and computational time for three different load levels (see Table 6).

From Table 6, IEPSO technique gives the best optimal solution with the lowest power losses, improved VSI and computational time as indicated in bold for all load conditions. The increased in load levels, gave results in increasing power losses and decreasing VSI.

For light load in case 2 IEPSO technique gave the most reduction in TPL by $64.69 \%, 11.34 \%$ highest VSI, but slightly defeated in computational time compared to IPSO by $0.7 \mathrm{~s}$ at light load. Meanwhile, for nominal load in case 2 IEPSO technique gave the most reduction for TPL by $73.06 \%, 26.14 \%$ highest VSI, but slightly defeated in computational time compared to IPSO by $0.98 \mathrm{~s}$ at nominal load. Finally, for heavy load in case 2 IEPSO technique gave the most reduction for TPL by $74.95 \%, 49.03 \%$ highest VSI, but slightly defeated in computational time compared to IPSO by $0.68 \mathrm{~s}$ at heavy load. 
Table 6. The performances of PSO, IPSO and IEPSO for DNR implementation in 69-bus radial distribution system.

\begin{tabular}{ccccc}
\hline \multirow{2}{*}{ Load Levels } & \multirow{2}{*}{ Parameters } & \multicolumn{3}{c}{ After Reconfiguration } \\
\cline { 2 - 5 } & & Case 2: PSO & Case 3: IPSO & Case 4: IEPSO \\
\hline \multirow{4}{*}{ Light Load } & Switches & $8,16,69,54,43$ & $14,20,8,9,69$ & $38,26,69,53,13$ \\
& TPL (kW) & 36.5 & 26.70 & 18.80 \\
& VSI min & 0.8423 & 0.8718 & 0.9298 \\
& Voltage profile (p.u.) & 0.9586 & 0.9663 & 0.9821 \\
& Computational time (s) & 44.078821 & 5.646365 & 6.350293 \\
\hline \multirow{4}{*}{ Nominal Load } & $45,16,53,8,69$ & $52,21,11,7,13$ & $9,13,61,52,19$ \\
& Switches & 145.5 & 69.0 & 61.9 \\
& TPL (kW) & 0.7102 & 0.7772 & 0.8576 \\
& VSI min & 0.9180 & 0.9389 & 0.9634 \\
& Voltage profile (p.u.) & 42.625656 & 6.981524 & 7.968446 \\
\hline \multirow{4}{*}{ Heavy Load } & $17,69,13,10,57$ & $13,5,59,69,58$ & $7,19,69,70,57$ \\
& Computational time (s) & 267.50 & 160.00 & 143.60 \\
& Switches & 0.6347 & 0.7401 & 0.7930 \\
& TPL (kW) & 0.8926 & 0.9348 & 0.9442 \\
& VSI min & 45.526328 & 7.059524 & 7.736560 \\
\hline
\end{tabular}

Table 7 summarizes the performances of three optimization techniques in DNR with DG sizing simultaneously for switches, TPL, VSI, voltage profile, DG sizing and computational time for three different load levels.

Table 7. The performances of PSO, IPSO and IEPSO for DNR with DG sizing implementation in 69-bus radial distribution system.

\begin{tabular}{ccccc}
\hline \multirow{2}{*}{ Load Levels } & Parameters & \multicolumn{3}{c}{ After Reconfiguration } \\
\cline { 2 - 5 } & & Case 2: PSO & Case 3: IPSO & Case 4: IEPSO \\
\hline \multirow{5}{*}{ Light Load } & Switches & $57,23,4,69,13$ & $19,57,45,10,12$ & $17,41,58,59,11$ \\
& DG sizing (MW) & 1.0100 & 1.0598 & 1.0911 \\
& TPL (kW) & 29.3 & 24.50 & 18.00 \\
& VSI min & 0.9205 & 0.9476 & 0.9649 \\
& Voltage profile min (p.u.) & 0.9801 & 0.9866 & 0.9911 \\
& Computational time (s) & 25.587512 & 6.312718 & 3.691640 \\
\hline \multirow{5}{*}{ Nominal Load } & Switches & $53,14,69,20$ & $55,12,14,9,16$ & $5,60,69,13,11$ \\
& DG sizing (MW) & 1.0930 & 1.0632 & 1.0376 \\
& TPL (kW) & 108.1 & 62.7 & 54.5 \\
& VSI min & 0.7356 & 0.8328 & 0.9137 \\
& Voltage profile min (p.u.) & 0.9349 & 0.9553 & 0.9777 \\
& Computational time (s) & 28.681047 & 7.874213 & 8.879116 \\
\hline \multirow{2}{*}{ Heavy Load } & Switches & $15,10,69,13$ & $22,18,69,7,54$ & $9,20,53,14,41$ \\
& DG & 1.0473 & 1.2238 & 1.0572 \\
& TPL (kW) & 237.3 & 138.30 & 118.90 \\
& VSI min & 0.6653 & 0.8127 & 0.8429 \\
& Voltage profile min (p.u.) & 0.9031 & 0.9502 & 0.9595 \\
& Computational time (s) & 35.443263 & 9.672387 & 5.418619 \\
\hline
\end{tabular}

From Table 7, the IEPSO technique gives the best optimal solution with the lowest power losses, improved VSI and computational time as indicated in bold for all load conditions. The increased in load levels, gave results in increasing power losses and decreasing VSI.

For light load in case 2 IEPSO technique gave the most reduction for TPL by $66.19 \%, 15.54 \%$ highest VSI, and the fastest in computational time compared to other techniques at light load. Meanwhile, 
for nominal load in case 2 IEPSO technique gave the most reduction for TPL by $76.28 \%, 34.39 \%$ highest VSI, but slightly defeated in computational time compared to IPSO by $1 \mathrm{~s}$ at nominal load. Finally, for heavy load in case 2 IEPSO technique gave the most reduction for TPL by $79.26 \%, 58.41 \%$ highest VSI, and the fastest in computational time compared to other techniques at heavy load.

Figures 8 and 9 show the stability is improved for all cases, especially when the proposed algorithm is applied on 69-bus test system. Generally, by increasing the load levels, the VSI will decrease. Thus, the stability of the system is towards the voltage collapse with increasing load demand.

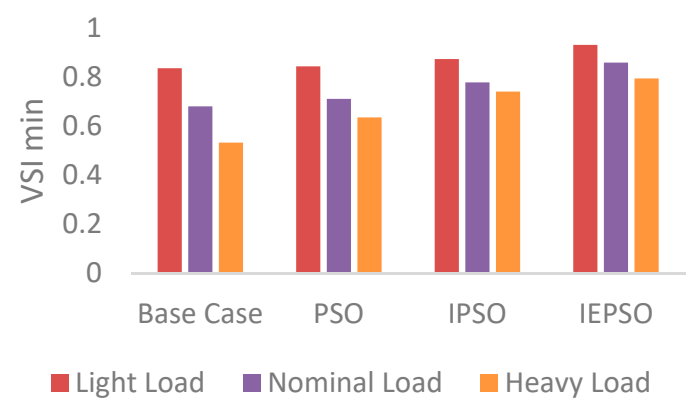

Figure 8. Voltage stability index for different load levels DNR in 69-bus test system.

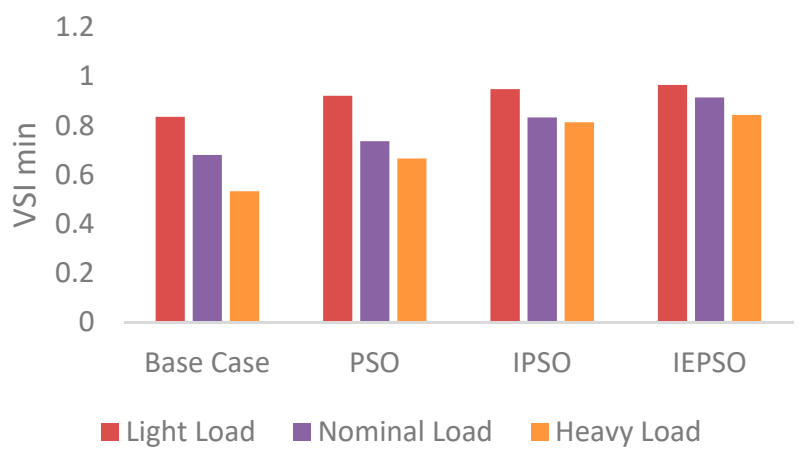

Figure 9. Voltage stability index for different load levels DNR with DG in 69-bus test system.

Figure 10 shows the comparison of TPL and VSI for three cases which are base case, DNR without DG and DNR with DG for light, nominal and heavy load condition in 69-bus system. From the figure, it is shown that TPL is slightly reduced with DNR implementation. However, the TPL has a significant reduced when DNR and DG was applied to the system.

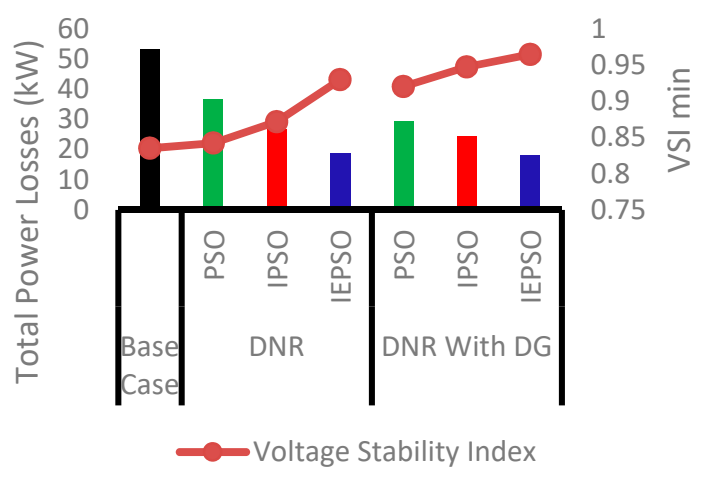

Figure 10. Total power losses and VSI min for DNR without and with DG at light load in 69-bus test system. 
At light load in 69-bus test system, the total power loss obtained from the PSO algorithm (case 2) with DG is $29.3 \mathrm{~kW}$ compared to $36.5 \mathrm{~kW}$ without DG with $7.2 \mathrm{~kW}$ differences, with $13.52 \%$ TPL reduction. The VSI without DG is 0.8423 which is improved to be 0.9205 with DG integration. For IPSO algorithm (case 3), the total power loss is $24.5 \mathrm{~kW}$ with DG compared to $26.7 \mathrm{~kW}$ without DG with $2.2 \mathrm{~kW}$ differences, with $4.13 \%$ TPL reduction. The VSI without DG is 0.8718 which is improved to be 0.9476 with DG integration. Finally, For IEPSO algorithm (case 4), the total power loss is $18 \mathrm{~kW}$ with DG compared to $18.8 \mathrm{~kW}$ without DG with $0.8 \mathrm{~kW}$ differences, with a $1.5 \%$ TPL reduction. The VSI without DG is 0.9298 which is improved to be 0.9649 with DG integration.

As for the last case, the total power loss obtained from the PSO algorithm (case 2) with DG is $108.1 \mathrm{~kW}$ compared to $145.5 \mathrm{~kW}$ without DG with $37.4 \mathrm{~kW}$ differences, with $16.28 \%$ TPL reduction, while, the VSI improved is by $3.74 \%$. For IPSO algorithm (case 3), the total power loss is $62.7 \mathrm{~kW}$ with DG compared to $69 \mathrm{~kW}$ without DG with $6.3 \mathrm{~kW}$ differences, with $2.74 \%$ TPL reduction, while, The VSI is improved by $8.18 \%$. Finally, For IEPSO algorithm (case 4), the total power loss is $54.5 \mathrm{~kW}$ with DG compared to $61.9 \mathrm{~kW}$ without DG with $7.4 \mathrm{~kW}$ differences, with $3.22 \%$ TPL reduction. The VSI is improved by $8.25 \%$.

Similarly, at heavy load in 69-bus test system, the total power loss obtained from the PSO algorithm (case 2) with DG is $237.3 \mathrm{~kW}$ compared to $267.5 \mathrm{~kW}$ without DG with $30.2 \mathrm{~kW}$ differences, with $5.27 \%$ TPL reduction. The VSI is improved by $5.75 \%$. For IPSO algorithm (case 3), the total power loss is $138.3 \mathrm{~kW}$ with DG compared to $160 \mathrm{~kW}$ without DG with $21.7 \mathrm{~kW}$ differences, with $3.79 \%$ TPL reduction. The VSI is improved by $13.64 \%$. For IEPSO algorithm (case 4), the total power loss is $118.9 \mathrm{~kW}$ with DG compared to $143.6 \mathrm{~kW}$ without DG with $24.7 \mathrm{~kW}$ differences, with $4.31 \%$ TPL reduction. The VSI is improved by $9.38 \%$.

From the results of both test systems, the following points can be summarized as below:

(i) the proposed IEPSO technique performed better results in terms of fitness function value and computational time as compared to PSO and IPSO techniques in terms of TPL reduction and VSI improvement as objective function as seen in Table 8.

(ii) the proposed IEPSO technique is effective in finding the optimal sizing of DG in distribution power systems.

(iii) the TPL reduction and VSI enhancement can be obtained after DG placement and sizing.

(iv) by increasing the load condition of the test systems, from light to heavy load, the TPL is found to be increased and VSI decreased. This is to say that as far as the system is having a heavier the load, the system is getting more unstable with the increment in losses.

(v) the voltage profiles are improved when DGs are installed at the optimal size and bus locations.

Table 8. Total power losses reduction and VSI improvement for IEPSO technique.

\begin{tabular}{|c|c|c|c|c|c|c|c|c|}
\hline \multirow{3}{*}{ Load Levels } & \multicolumn{4}{|c|}{ 33-Bus System } & \multicolumn{4}{|c|}{ 69-Bus System } \\
\hline & \multicolumn{2}{|c|}{ DNR } & \multicolumn{2}{|c|}{ DNR with DG } & \multicolumn{2}{|c|}{ DNR } & \multicolumn{2}{|c|}{ DNR with DG } \\
\hline & $\begin{array}{c}\text { TPL } \\
\text { Reduction } \\
(\%)\end{array}$ & $\begin{array}{c}\text { VSI } \\
\text { Improvement } \\
(\%)\end{array}$ & $\begin{array}{c}\text { TPL } \\
\text { Reduction } \\
(\%)\end{array}$ & $\begin{array}{c}\text { VSI } \\
\text { Improvement } \\
(\%)\end{array}$ & $\begin{array}{c}\text { TPL } \\
\text { Reduction } \\
(\%)\end{array}$ & $\begin{array}{c}\text { VSI } \\
\text { Improvement } \\
(\%)\end{array}$ & $\begin{array}{c}\text { TPL } \\
\text { Reduction } \\
(\%)\end{array}$ & $\begin{array}{c}\text { VSI } \\
\text { Improvement } \\
(\%)\end{array}$ \\
\hline Light Load & 4.16 & 2.62 & 17.98 & 10.34 & 64.69 & 11.34 & 66.19 & 15.54 \\
\hline Nominal Load & 23.26 & 5.21 & 36.33 & 18.16 & 73.06 & 26.14 & 76.28 & 34.39 \\
\hline Heavy Load & 31.36 & 21.14 & 37.73 & 28.22 & 74.95 & 49.03 & 79.26 & 58.41 \\
\hline
\end{tabular}

\section{Conclusions}

In this research an effective heuristic optimization technique called IEPSO was proposed. The aim of this optimization function is to solve three optimization problems. The first problem was to determine optimal DG location. Meanwhile, the second optimization problem was to minimize TPL and VSI with DNR implementation. Finally, the third issue was to get the minimum TPL and better VSI with DNR and DG implementation. Thus, the objective function was formulated to minimize TPL and to improve the VSI. VSI is determined for three load demand levels namely light load, nominal 
load, and heavy load with proper optimal DNR and DG sizing. The performance of the proposed technique was compared with other optimization techniques, namely PSO and IPSO. Four case studies on IEEE 33-bus and IEEE 69-bus distribution systems have been conducted to validate the effectiveness of the proposed IEPSO. The optimization results show that, the best achievement is done by IEPSO technique with power losses reduction up to $79.26 \%$, and $58.41 \%$ improvement in the voltage stability index. Moreover, IEPSO has the fastest computational time for all load conditions as compared to other algorithms.

Author Contributions: N.F.N., A.F.A.K., E.E.H., M.F.S. and T.K. have done the Methodology, Simulation, Validation, Analysis, and wrote the paper. Conceptualization, N.F.N., A.F.A.K., E.E.H., M.F.S. and T.K.; Methodology, N.F.N., A.F.A.K., E.E.H., M.F.S. and T.K.; Software, N.F.N., A.F.A.K., E.E.H., M.F.S. and T.K.; Validation, N.F.N., A.F.A.K., E.E.H., M.F.S. and T.K., and Z.Z.; Formal Analysis, N.F.N., A.F.A.K., E.E.H., M.F.S. and T.K.; Investigation, N.F.N., A.F.A.K., E.E.H., M.F.S. and T.K.; Resources, N.F.N., A.F.A.K., E.E.H., M.F.S. and T.K.; Data Curation, N.F.N., A.F.A.K., E.E.H., M.F.S. and T.K.; Writing-Original Draft Preparation, N.F.N., A.F.A.K., E.E.H., M.F.S. and T.K.; Writing-Review \& Editing, N.F.N., A.F.A.K., E.E.H., M.F.S. and T.K.; Visualization, N.F.N., A.F.A.K., E.E.H., M.F.S. and T.K.; Supervision, N.F.N., A.F.A.K., E.E.H., M.F.S. and T.K.; Project Administration, N.F.N., A.F.A.K., E.E.H., M.F.S. and T.K.; Funding Acquisition, N.F.N., A.F.A.K., E.E.H., M.F.S. and T.K.

Funding: This research was partially funded by An-Najah National University and Palestine Ministry of Higher Education grant number ANNU-MoHE-1819-Sc003 and grant number ANNU-1718-Sc0014. Financial support is also received form Palestinian Universities Support Fund, Grant No1458959.

Conflicts of Interest: The authors declare no conflict of interest.

\section{References}

1. Al-abri, R. Voltage Stability Analysis with High Distributed Generation (DG) Penetration. Ph.D. Thesis, University of Waterloo, Waterloo, ON, Canada, 2012.

2. Abdul Kadir, A.; Khatib, T.; Elmenreich, W. Integrating photovoltaic systems in power system: Power quality impacts and optimal planning challenges. Int. J. Photoenergy 2014, 2014, 321826. [CrossRef]

3. Dondi, P.; Bayoumi, D.; Haederli, C.; Julian, D.; Suter, M. Network integration of distributed power generation. J. Power Sources 2002, 106, 1-9. [CrossRef]

4. Masters, C.L. Voltage rise: The big issue when connecting embedded generation to long $11 \mathrm{kV}$ overhead lines. Power Eng. J. 2002, 16, 5-12. [CrossRef]

5. Chuong, T.T. Distributed Generation Impact on Voltage Stability in Distribution Networks. System 2008, $25,1-5$.

6. Hedayati, H.; Nabaviniaki, S.A.; Akbarimajd, A. A Method for Placement of DG Units in Distribution Networks. IEEE Trans. Power Deliv. 2008, 23, 1620-1628. [CrossRef]

7. Gil, H.A.; el Chehaly, M.; Joos, G.; Cañizares, C. Bus-based indices for assessing the contribution of DG to the voltage security margin of the transmission grid. In Proceedings of the 2009 IEEE Power Energy Society General Meeting PES '09, Calgary, AB, Canada, 26-30 July 2009; pp. 1-7.

8. Paliwal, P.; Patidar, N.P.; Nema, R.K. A Comprehensive Survey of Optimization Techniques used for Distributed Generator Siting and Sizing. In Proceedings of the 2012 IEEE Southeastcon, Orlando, FL, USA, 15-18 March 2012.

9. Jamian, J.J.; Abdullah, M.N.; Mokhlis, H.; Mustafa, M.W.; Bakar, A.H.A. Global particle swarm optimization for high dimension numerical functions analysis. J. Appl. Math. 2014, 2014, 329193. [CrossRef]

10. Chen, H.; Chen, J.; Shi, D.; Duan, X. Power flow study and voltage stability analysis for distribution systems with distributed generation. In Proceedings of the IEEE Power Engineering Society General Meeting, Montreal, QC, Canada, 18-22 June 2006.

11. Alonso, M.; Amarís, H. Voltage stability in distribution networks with DG. In Proceedings of the 2009 IEEE Bucharest PowerTech, Bucharest, Romania, 28 June-2 July 2009; pp. 1-6.

12. Grigsby, L.L. The Electric Power Engineering Handbook; CRC Press: Boca Raton, FL, USA, 2001; Volume 2.

13. Slootweg, J.G.; Kling, W.L. Impacts of distributed generation on power system transient stability. In Proceedings of the IEEE Power Engineering Society Summer Meeting, Chicago, IL, USA, 21-25 July 2002.

14. Reza, M.; Schavemaker, P.H.; Slootweg, J.G.; Kling, W.L.; van der Sluis, L. Impacts of distributed generation penetration levels on power systems transient stability. In Proceedings of the IEEE Power Engineering Society General Meeting, Denver, CO, USA, 6-10 June 2004. 
15. Freitas, W.; Vieira, J.C.M.; da Suva, L.C.P.; Affonso, C.M.; Morelato, A. Long-term voltage stability of distribution systems with induction generators. In Proceedings of the IEEE Power Engineering Society General Meeting, San Francisco, CA, USA, 16 June 2005; pp. 3-6.

16. Freitas, W.; Morelato, A.; Xu, W.; Sato, F. Impacts of AC generators and DSTATCOM devices on the dynamic performance of distribution systems. In IEEE Transactions on Power Delivery; IEEE: Piscataway Township, NJ, USA, 2005; Volume 20, pp. 1493-1501.

17. Freitas, W.; da Silva, L.C.P.; Morelato, A. Systems With Induction Generators. Power 2005, 20, 1653-1654.

18. Kotamarty, S.; Khushalani, S.; Schulz, N. Impact of distributed generation on distribution contingency analysis. Electr. Power Syst. Res. 2008, 78, 1537-1545. [CrossRef]

19. Londero, R.R.; Affonso, C.M.; Nunes, M.V.A. Impact of distributed generation in steady state, voltage and transient stability. In Proceedings of the IEEE Bucharest PowerTech, Bucharest, Romania, 28 June-2 July 2009; pp. 1-6.

20. Jasmon, G.B.; Lee, L.H.C.C. New Contingency Ranking Technique Incorporating a Voltage Stability Criterion. IEE Proc.-C 1993, 140, 87-90. [CrossRef]

21. Kashem, M.A.; Ganapathy, V.; Jasmon, G.B. Network reconfiguration for enhancement of voltage stability in distribution networks. IEE Proc. Gener. Transm. Distrib. 2000, 147, 171. [CrossRef]

22. Shin, J.-R.; Kim, B.-S.; Park, J.-B.; Lee, K.Y. A New Optimal Routing Algorithm for Loss Minimization and Voltage Stability Improvement in Radial Power Systems. IEEE Trans. Power Syst. 2007, 22, 648-657. [CrossRef]

23. Aman, M.M.; Jasmon, G.B.; Bakar, A.H.A.; Mokhlis, H. A new approach for optimum DG placement and sizing based on voltage stability maximization and minimization of power losses. Energy Convers. Manag. 2013, 70, 202-210. [CrossRef]

24. Esmaili, M.; Firozjaee, E.C.; Shayanfar, H.A. Optimal placement of distributed generations considering voltage stability and power losses with observing voltage-related constraints. Appl. Energy 2014, 113, 1252-1260. [CrossRef]

25. Esmaili, M. Placement of minimum distributed generation units observing power losses and voltage stability with network constraints. IET Gener. Transm. Distrib. 2013, 7, 813-821. [CrossRef]

26. Moradi, M.H.; Tousi, S.M.R.; Abedini, M. Multi-objective PFDE algorithm for solving the optimal siting and sizing problem of multiple DG sources. Int. J. Electr. Power Energy Syst. 2014, 56, 117-126. [CrossRef]

27. Lee, T.Y.; Chen, C.L. Unit commitment with probabilistic reserve: An IPSO approach. Energy Convers. Manag. 2007, 48, 486-493. [CrossRef]

28. Chakravorty, M.; Das, D. Voltage stability analysis of radial distribution networks. Int. J. Electr. Power Energy Syst. 2001, 23, 129-135. [CrossRef] 